\title{
Numerical investigation of the flow field in the upper human airways
}

\author{
G. Eitel, W. Schröder \& M. Meinke \\ Institute of Aerodynamics, RWTH Aachen University, \\ Wüllnerstrasse 5a, 52062 Aachen, Germany
}

\begin{abstract}
The flow in a realistic model of the human lung is numerically simulated at steady and unsteady inspiration and expiration. A model of a human lung ranging from the trachea down to the sixth generation of the bronchial tree is used for the simulation. The numerical analysis is based on the Lattice-Boltzmann method, which is particularly suited for flows in extremely intricate geometries such as the upper human airways.

The results for steady air flow at inspiration and expiration for a diameter based Reynolds number of $R e_{D}=1250$ evidence secondary vortex structures and air exchange mechanisms. It is shown that the asymmetric geometry of the human lung plays a significant role for the development of the flow field in the respiratory system. Secondary vortex structures observed in former studies are reproduced and described in detail.

The solutions for unsteady respiration allow a detailed analysis of the temporal formation of secondary flow structures whereas the time dependence is much more pronounced at inspiration than at expiration.

Keywords: computational fluid dynamics, Lattice-Boltzmann method, respiratory system, human lung, unsteady flow.
\end{abstract}

\section{Introduction}

The human lung is a complex respiratory system consisting of a repeatedly bifurcating network of tubes with progressively decreasing diameters. The understanding of the flow processes in the upper human airways is of great importance in developing aerosol drug delivery systems and to improve the efficiency and usability of artificial respiration. Numerous experimental and numerical investigations of 
lung flow have been conducted so far [1-5]. However, due to the high geometric intricacy of the human lung, there is still a considerable amount of uncertainty concerning the very complex flow field and results for realistic lung models are still rare. Most of the investigations are based on simplified models of the lung structure. The most popular of these models is the so-called Weibel model [6], which describes the bronchi as a symmetric tree structure of subsequent bifurcating tubes with 23 generations. However, in most of the studies only the first three to five generations are considered and a planar representation is often favored for simplicity. In [7], detailed experimental studies were performed for a planar Weibel model where fundamental flow phenomena, such as m-shaped velocity profiles and counter-rotating vortices, were described. It was shown in numerical studies $[1,8]$, however, that the flow field for the non-planar configuration differs significantly from the planar case. Studies considering asymmetric bifurcations [9], non-smooth surfaces [10] and CT-based models $[2,5,11]$ show that the inspiratory flow in the upper human airways is asymmetric and swirling and the results emphasize the importance of realistic airway models. Generally, the aforementioned results show that an accurate lung geometry, i.e., CT data or a real human lung cast, is required to obtain physically relevant results.

However, when numerical simulations are considered the accuracy does not only depend on the geometry, but also on the quality of the numerical method, the computational resolution, and the boundary conditions. Therefore, it is necessary to validate the numerical solutions either by experimental results or by a detailed comparison with existing numerical data from the literature or by proving the quality of the method through an analysis of a similar, well established flow problem.

The present work focuses on the detailed investigation of the three-dimensional flow in a realistic model of the human based on an actual lung cast. The geometry covers the trachea and the bronchial tree down to the sixth generation. A silicon model of the same geometry has been experimentally investigated in [3]. The flow field is simulated via the Lattice-Boltzmann method (LBM) [12]. Unlike former numerical and experimental investigations, in which a simplified geometry was used, the present method can be efficiently applied to variable, realistic airway geometries. For instance the flow field downstream of the laryngeal region has recently been investigated in [13] by an LBM. Since the numerical method is capable of reproducing small-scale features of lung flow, the results serve to fundamentally understand respiratory mechanisms. Thus, the numerical results allow an extended analysis of the three-dimensional flow structures observed in [3].

The steady flow field at inspiration and expiration has been simulated for a constant Reynolds number based on the hydraulic diameter of the trachea $D$ of $R e_{D}=1250$. Furthermore, the flow field at time dependent inhalation and exhalation has been computed with a peak Reynolds number of $R e_{D}=1050$ and a Womersley number of $\alpha=3.27$, where the Womersley number is defined as $\alpha=0.5 D \sqrt{2 \pi f / v}$ and $f$ is the frequency of the respiratory cycle and $v$ is the kinematic viscosity of air. The results mainly serve to fundamentally understand 
the three-dimensional flow structures within the upper bifurcations of the human lung under normal breathing conditions and the time-dependent development of the flow field.

The structure of this paper is as follows. First, the numerical method is briefly described. Then, the results for steady air flow are presented and compared with experimental data. Subsequently, the temporal development of secondary flow structures are discussed. Finally, the findings are summarized and some conclusions are drawn.

\section{The Lattice-Boltzmann method}

In the following, a concise description of the Lattice-Boltzmann Method (LBM) using the Bhatnagar, Gross, and Krook (BGK) [14] approximation will be given. A detailed derivation of the LBM and an extensive discussion can be found in [12]. The BGK approximation uses a simplified collision term for the Boltzmann equation leading to the so-called BGK equation without external forcing

$$
\frac{\partial f}{\partial t}+\xi_{i} \cdot \frac{\partial f}{\partial x_{i}}=\omega\left(f^{e q}-f\right) .
$$

The quantity $\omega$ represents the collision frequency, $f^{e q}$ is the Maxwell equilibrium distribution function, $f$ is the particle distribution function, and $\xi_{i}$ is the $i$-th component of the molecular velocity vector. That is, the left-hand side of Eq. 1 contains the temporal change and the propagation term, whereas the right-hand side describes molecular collisions. The corresponding algorithm is based on the iterative computation of propagation and collision processes for each cell of the computational grid. The macroscopic flow variables are determined by summation over the base moments of the distribution function $f$.

Since the LBM formulation is based on a uniform Cartesian grid, it is highly adapted for parallel computation and it offers an efficient boundary treatment for fixed walls. The computational grid is automatically generated from arbitrary surface data by an in-house grid generator [15]. The ability of reproducing variable organic geometries makes this method well suited for biomedical applications. The standard LBM describes weakly compressible flows and it has been shown in the literature [16] that the LBM yields indeed solutions to the Navier-Stokes equations. All results presented in this study for the incompressible flow in the upper human airways have been obtained by the Lattice Boltzmann method using the BGK equation (LBGK) method. More details on the numerical approach can be found in $[17,18]$.

\section{Results}

\subsection{Steady flow field}

To understand the global structure of the flow field, simulations have been performed for steady inspiration and expiration at a constant Reynolds number 


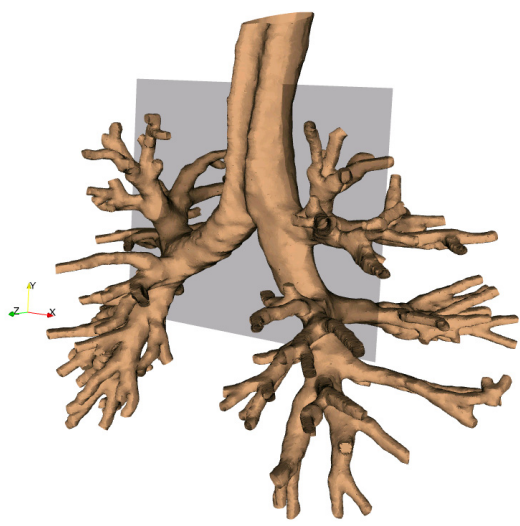

(a)

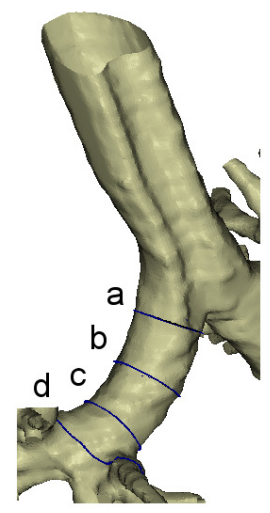

(b)

Figure 1: Reference plane to compare experimental and numerical data (left) and definition of the location of the cross sections for the investigation of secondary flow structures in the left primary bronchus (right).

of $R e_{D}=1250$. The value for $R e_{D}$ is based on the hydraulic diameter of the throat and corresponds to a mass flux of $240 \mathrm{ml} / \mathrm{s}$. A Dirichlet condition, i.e., the velocity distribution, has been imposed at the tracheal cross section at inspiration and expiration. To validate the numerical solutions, the results are compared with experimental data obtained by Particle Imaging Velocimetry (PIV) measurements, which have been performed using a silicon model of the same lung geometry [3]. The results are investigated at a reference plane the location of which is indicated in Fig. 1(a).

The comparison of the velocity magnitude contours at inspiration in Fig. 2 shows good agreement between experiment and simulation.

The numerical bulk velocity distribution in the tracheal section is nearly identical with the experimental profile and the decomposition of the mass flux in the left and right primary bronchus is very similar. The velocity contours at the upper left primary bronchus, which indicate a recirculation region with counterrotating vortices whose axes lie in the stream-wise direction, are also in good agreement.

At expiration (Fig. 3) the numerical and experimental data for the velocity contours are also alike, although the velocity magnitude in the numerical solution is smaller than that in the measurements.

The velocity distribution in the tracheal region appears to be more developed in the experimental case. This slight difference is likely to be due to the boundary 

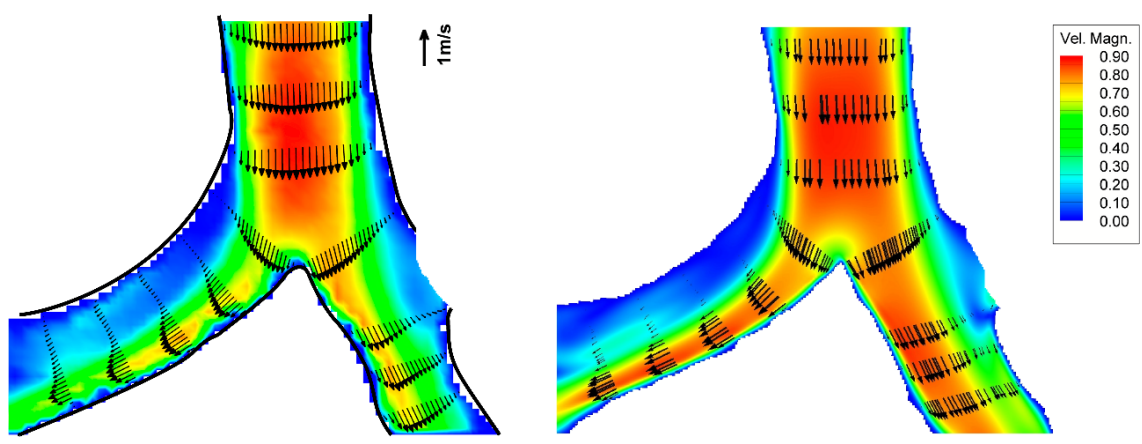

Figure 2: Velocity contours and distributions at inspiration at $R e_{D}=1250$; PIV (left), LBM (right).
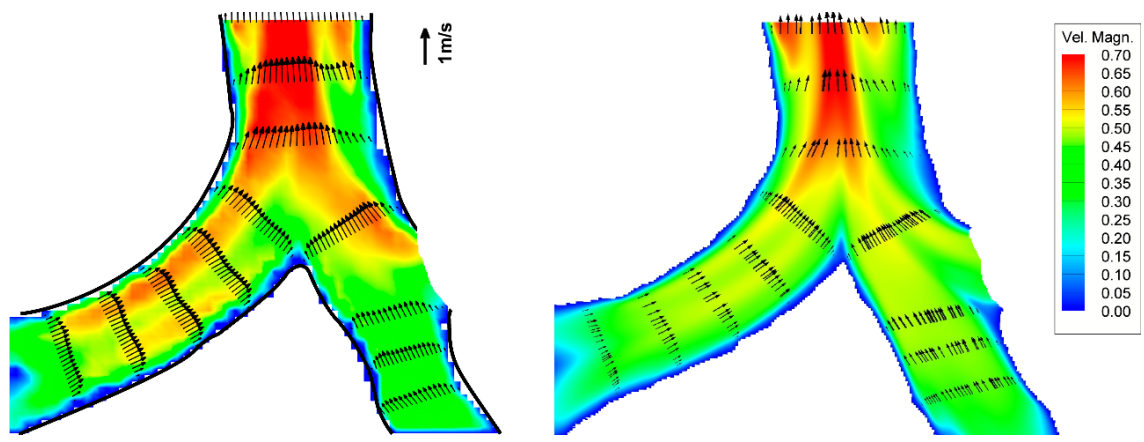

Figure 3: Velocity contours and distributions at expiration at $R e_{D}=1250$; PIV (left), LBM (right).

conditions at the outlet cross sections of the highest generations of the bronchial tree. In the experiment these cross sections were accessed by drilling holes in the silicon body for mass feeding or discharge, respectively. In the simulation a vanishing velocity gradient has been prescribed at inspiration and expiration.

The current numerical method allows a detailed investigation of secondary flow structures in the primary bronchi. These flow structures have been observed in other numerical studies $[5,8,9]$ and in experimental findings [3, 19]. The velocity distributions and contours in the left primary bronchus at four cross sections described in Fig. 1(b) are shown in Fig. 4.

At inspiration Fig. 4 shows that the main mass flux is located near the lower wall indicated by a high stream-wise velocity. Downstream of the first bifurcation a pair of counter-rotating vortices develops in which air is transported away from the high speed region along the outer walls. The left vortex has a center of rotation near to the upper wall and fills up nearly the upper half of the cross section. The right vortex is much smaller and is located very close to the right wall. The vortex pair has also been observed in the experiment [3]. Additionally, the analysis of 
a

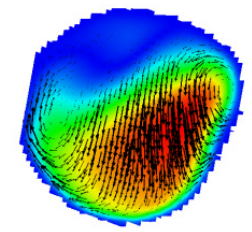

b

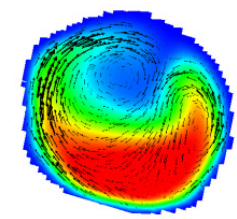

C
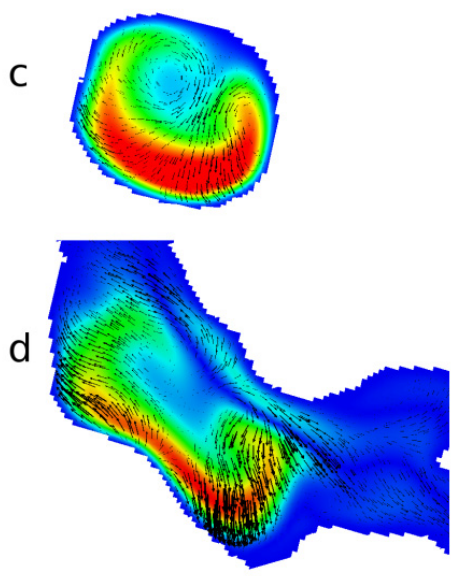

a

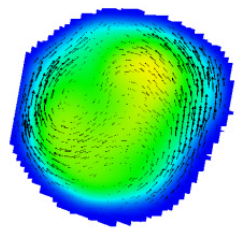

Vel.

Magn.

b

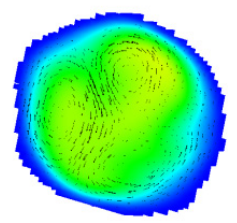

$/ \mathrm{m} / \mathrm{s}$

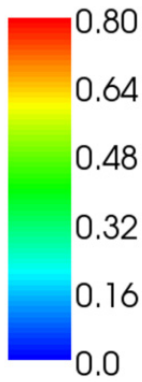

Figure 4: In-plane velocity distributions (arrows) and axial velocity contours (shades of gray) in several cross sections defined in Fig. 1(b) in the left principal bronchus at steady inspiration (left) and steady expiration (right).

the numerical data emphasizes the strong asymmetry of the vortical structures. When the next bifurcation is reached, the vortices do separate and each one enters a branch of the next bronchial generation as shown in Fig. 4(d) at inspiration.

At expiration the stream-wise velocity is fully distributed and the indicated vortical structures clearly possess a much smaller ratio of azimuthal momentum to stream-wise momentum than at expiration. The mixing of two streams coming from the higher generation bronchi generates a shear layer which develops into a slightly swirling region in Fig. 4(c). In Fig. 4(a) an in-plane velocity, pointing upwards, is evidenced near the walls. In conclusion, the findings of the steady flow 


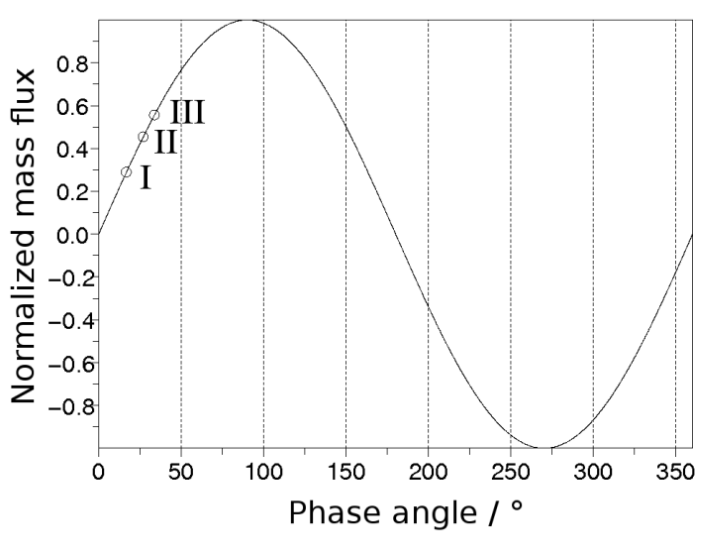

Figure 5: Modeling of the unsteady flow by a sinusoidal distribution of the mass flux.

field confirm that much more secondary flow structures are generated at inspiration than at expiration.

\subsection{Unsteady flow field}

In order to investigate the temporal development of the secondary flow structures, extensive simulations concerning the unsteady behavior of the flow field during oscillating respiratory ventilation have been performed. The results evidence that the temporal rate of change of the velocity has a strong impact on the development of the flow field in the bifurcations. Simulations of oscillating flows have been conducted for a $R e_{D}=1050$ and a $\alpha=3.27$ corresponding to a time period of $T=3.7 \mathrm{~s}$ which describes a normal respiration at rest. The temporal change of the mass flux has been prescribed by a sinusoidal curve shown in Fig. 5. The data acquisition has been started after a transient time of two respiration cycles.

The velocity distributions for a whole respiration cycle are shown in Fig. 6 for the trachea and the first bifurcation.

When the mass flux peaks, the flow field is almost identical with the steady case distribution. At a phase angle of $45^{\circ}$ the overall flow structures are already fully developed. However, the high speed region in the left primary bronchus still becomes more narrow at increasing phase angle. At expiration the overall flow structure does not appear to change noticeably in time. The comparison with the time dependent PIV findings from [3] shows the temporal behavior of the numerically determined flow field to be in good agreement with the experimental results.

In order to obtain insight into the development of secondary flow structures at inspiration, the flow at the very beginning of inhalation, i.e., at very low Reynolds 

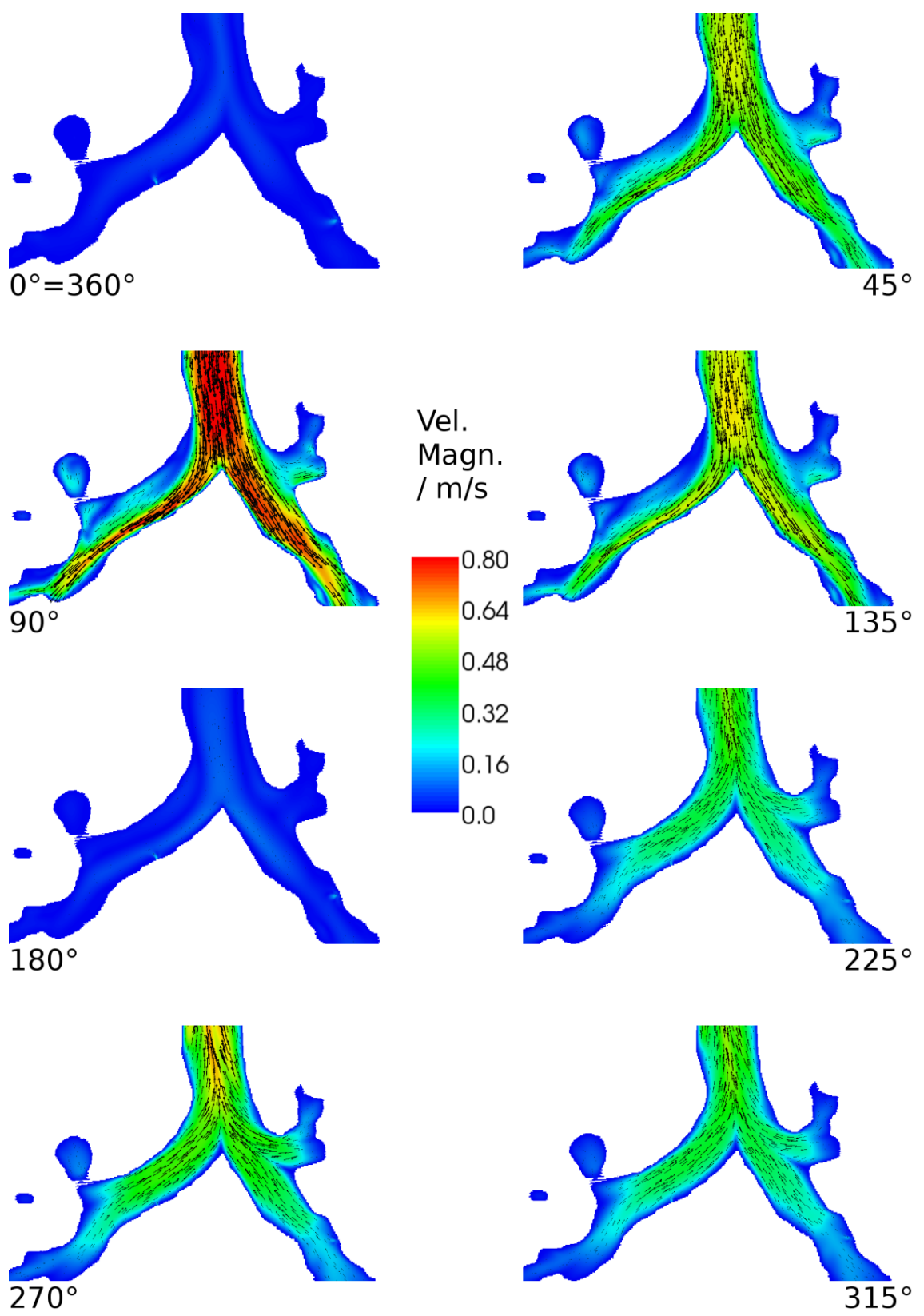

Figure 6: Velocity distributions at unsteady simulation. The numbers indicate the phase angles depicted in Fig. 5. 


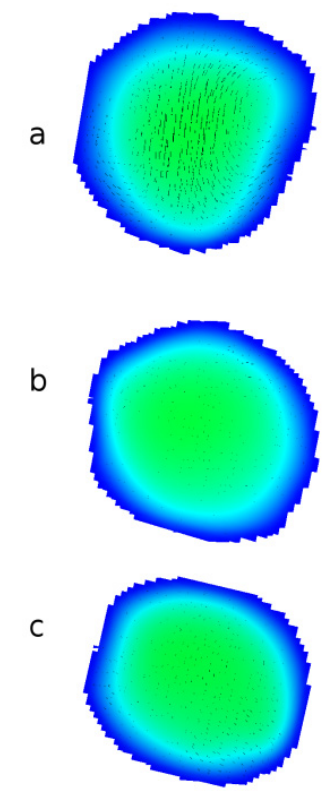

I.
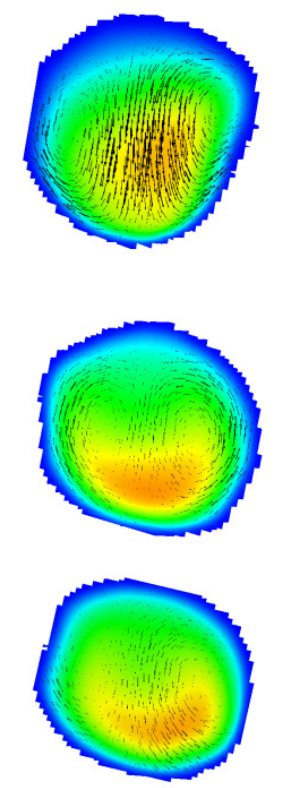

II.
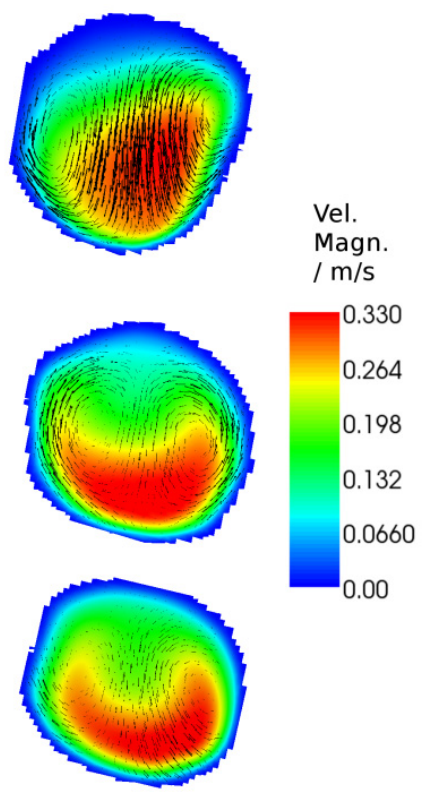

III.

Figure 7: In-plane velocity distributions (arrows) and axial velocity contours (shades of gray) for three cross sections depicted in Fig. 1(b). The numbers indicate three subsequent time steps as shown in Fig. 5.

numbers, has been investigated. In Fig. 7 the temporal evolution of the flow field in the left primary bronchus is shown for three time values indicated in Fig. 5.

The observed sequence starts $173 \mathrm{~ms}$ after the bulk velocity at the inlet cross section has reversed. In Fig. 7(I) the distribution of the axial velocity shows a rather flat pattern and no vortical structures are visible. The flow field is very similar in all three cross sections. The second time step depicted in Fig. 7(II) shows an increasing axial velocity and an incipient asymmetry of the axial velocity distribution, i.e., the location of the peak value occurs close to the lower wall. This development is enhanced in the stream-wise direction. The in-plane velocity distribution evidences the formation of counter rotating vortical structures throughout the cross sections. In the last stage of the analysis shown in Fig. 7(III) both the counter-rotating vortices and the axial velocity distribution are very similar to those observed at maximum inspiration in all three cross sections.

The numerical results show that the elementary vortical structures and the high speed region which have been observed at maximum inspiration are already encountered at a phase angle of $34^{\circ}$ corresponding to a Reynolds number of 
$R e_{D}=590$. This is agreement with the experiment where the size of the counterrotating vortices has been found to be Reynolds number independent as long as the Reynolds number is above a critical level.

\section{Conclusions}

The flow field in a realistic model of the human lung at steady inspiration and expiration and at unsteady respiration has been simulated via the LBM. The LBM has proved to be an efficient tool to simulate flows through highly intricate geometries which have been resolved by an automatically generated Cartesian mesh.

The steady flow field at inspiration and expiration has been analyzed for a constant flow rate of $240 \mathrm{ml} / \mathrm{s}$ resulting in a Reynolds number based on the hydraulic diameter of the throat region of $\operatorname{Re}_{D}=1250$. The visualization has evidenced the intricate three-dimensional character of the flow field. A pair of counter-rotating vortices and a region of high speed flow have been observed downstream of the first bifurcation in the left bronchus. The results have been compared with PIV measurements and showed to be in very good agreement with the experimental findings. Furthermore, the separation of the vortices in the next bifurcation and a strong asymmetry have been observed.

In order to evidence the impact of an oscillating mass flux the unsteady flow field has been analyzed at a Womersley number of $\alpha=3.27$ and a maximum Reynolds number of $R e_{D}=1050$. In a preliminary analysis the overall flow structure has been shown to be similar to that of the steady case. The growth of the vortical structures in the left branch of the first bifurcation has been investigated in detail for initiating inspiration. The numerical data evidences a strong dependence of the shape and size of the secondary flow structures on the instantaneous mass flux. At Reynolds numbers greater than $R e_{D}=600$ the overall shape of the flow field does not change. At expiration the steady and unsteady flow solutions are found to be very similar since hardly any secondary flow structures have been observed.

The obtained results reveal insight into the overall structure of the flow field in a realistic lung geometry and emphasize the unsteady character of the flow field when the flow conditions reverse. This knowledge is essential for the improvement of artificial respiration devices and for the development of aerosol drug delivery systems.

\section{Acknowledgement}

The support of the Deutsche Forschungsgemeinschaft (DFG) is gratefully acknowledged. 


\section{References}

[1] Comer, J.K., Kleinstreuer, C. \& Kim, C.S., Flow structures and particle desposition patterns in double-bifurcation airway models. Part 1. Air flow fields. 435, pp. 25 - 54, 2001.

[2] Nowak, N., Kakade, P.P. \& Annapragada, A.V., Computational Fluid Dynamics Simulation of Airfoil and Aerosol Deposition in Human lungs. Annals of Biomedical Eng, 31, pp. 374 - 390, 2003.

[3] Große, S., Schröder, W., Klaas, M., Klöckner, A. \& Roggenkamp, J., Time resolved analysis of steady and oscillating flow in the upper human airways. Experiments in Fluids, 42, pp. 955-970, 2007.

[4] Große, S., Schröder, W. \& Klaas, M., Time-Resolved PIV Measurements of Vortical Structures in the Upper Human Airways. Particle Image Velocimetry, Springer Berlin/Heidelberg, volume 112/2008 of Topics in Applied Physics, pp. 35-53, 2008.

[5] van Ertbruggen, C., Hirsch, C. \& Paiva, M., Anatomically based threedimensional model of airways to simulate flow and particle transport using computational fluid dynamics. J Appl Physiol, 98, pp. 970-980, 2005.

[6] Weibel, E., Morphometry of the human Lung. Springer Berlin, 1963.

[7] Zhao, Y. \& Lieber, B.B., Steady Expiratory Flow in a Model Symmetric Bifurcation. Journal of Biomechanical Engineering, 116(3), pp. 318-323, 1994.

[8] Zhang, Z., Kleinstreuer, C. \& Kim, C.S., Gas-solid two-phase flow in a triple bifurcation lung airway model. International Journal of Multiphase Flow, 28(6), pp. 1021 - 1046, 2002.

[9] Liu, Y., So, R.M.C. \& Zhang, C.H., Modelling the bifurcating flow in a human lung airway. Journal of Biomechanics, 35(4), pp. 465-473, 2003.

[10] Li, Z., Kleinstreuer, C. \& Zhang, Z., Particle deposition in the human tracheobronchial airways due to transient inspiratory flow patterns. Journal of Aerosol Science, 38(6), pp. 625 - 644, 2007.

[11] Lin, C.L., Tawhai, M.H., McLennan, G. \& Hoffman, E.A., Characteristics of the turbulent laryngeal jet and its effect on airflow in the human intra-thoracic airways. Respiratory Physiology \& Neurobiology, 157(2-3), pp. 295 - 309, 2007.

[12] Benzi, R., Succi, S. \& Vergassola, M., The Lattice Boltzmann Equation: Theory and Applications. Physics Reports, 222(No. 3), pp. 145-197, 1992.

[13] Ball, C.G., Uddin, M. \& Pollard, A., Mean flow structures inside the human upper airway. Flow, Turbulence and Combustion, 81, pp. 155-188, 2008.

[14] Bhatnagar, P.L., Gross, E.P. \& Krook, M., A Model for Collision Processes in Gases. I. Small Amplitude Processes in Charged and Neutral OneComponent Systems. Phys Rev, 94(3), pp. 511-525, 1954.

[15] Hartmann, D., Meinke, M. \& Schröder, W., An adaptive multilevel multigrid formulation for Cartesian hierarchical grid methods. Comput Fluids, 37, pp. 1103-1125, 2008.

[16] Hänel, D., Molekulare Gasdynamik. Springer: Berlin, 2004. 
114 Modelling in Medicine and Biology VIII

[17] Freitas, R.K. \& Schröder, W., Numerical investigation of the threedimensional flow in a human lung model. Journal of Biomechanics, 2008.

[18] Freitas, R.K., Meinke, M. \& Schröder, W., Investigation of Wall-Bounded Turbulent Flow using Lattice Boltzmann Methods. submitted to Computers \& Fluids, 2008.

[19] Adler, K. \& Brücker, C., Dynamic flow in a realistic model of the upper human lung airways. Experiments in Fluids, 43(2), pp. 411-423, 2007. 